\title{
A note on the Maxwell Equations of electromagnetic irradiation in a holed spatial region
}

\section{Abstract}

We make relevant and important comments on the electromagnetic irradiation in a holed spatial region by writing the Maxwell equations on light of the Helmoholtz Theorem for vectorial fields on $\mathbb{R}^{3}$.

Keywords: electromagnetic irradiation, Helmholtz theorem, landau Gauge, propagator
Volume 3 Issue I - 2019

\section{Luiz CL Botelho}

Department of Applied Mathematics, Mathematics Institute, Fluminense Federal University, Brazil

Correspondence: Luiz CL Botelho, Department of Applied Mathematics, Mathematics Institute, Fluminense Federal University, Rua Mario Santos Braga, CEP 24220-140 Niterói, Rio de Janeiro, Brazil, Email botelho.luiz@ig.com.br

Received: October 23, 2018 | Published: January II, 2019
The non space-time approach for electromagnetic irradiation in a holed spatial region-Maxwell equations

One important point to be fully kept in mind when analyzing electromagnetic irradiation at a non quantum mechanical level is to consider as first and fundamental dynamical equations describing the phenomena of electromagnetic irradiation the first order system of Partial Differential Equations for the physical (measurable) strenght electromagnetic field; the full Maxwell equations on the vacuum written below:

$$
\begin{gathered}
\vec{\nabla} \cdot \vec{E}=4 \pi \rho \\
\vec{\nabla} \times \vec{H}=\frac{4 \pi}{c} \vec{J}_{E}+\frac{1}{c} \frac{\partial}{\partial t} \vec{E} \\
\operatorname{div} \vec{H}=0 \\
\vec{H} \times \vec{E}+\frac{1}{c} \frac{\partial \vec{H}}{\partial t}=0
\end{gathered}
$$

In classical electrodynamics and in the situation of the existence of compact holes on the $\mathbb{R}^{3}$, one must apply the Helmoholtz theorem first for the vector field $(\vec{E}, \vec{H})$, before writing the dynamical wave equations for the potentials, as we have earlier. ${ }^{1}$

Namely by a direct application of the Helmoholtz theorem to the magnetic vector field:

$$
\vec{H}=\left(\vec{\nabla} \times \vec{H}_{H}\right)+\left(\operatorname{grad} \phi_{H}\right)+\vec{H}^{\text {top }}
$$

where $\vec{H}^{\text {top }}$ is the unrotational harmonic magnetic topological field piece and $\phi_{H}$ the mag- netic scalar potential suppose from here on to be given explicitly as boundary conditions. On basis of the Helmoholtz theorem for the magnetic vector field eq(2), one has the identity for eq(1d)

$$
\vec{\nabla} \times\left(\vec{E}+\frac{1}{c} \frac{\partial \vec{A}_{H}}{\partial t}\right)=\left(-\frac{1}{c} \frac{\partial}{\partial t} \operatorname{grad} \phi_{H}\right)-\frac{1}{c} \frac{\partial \vec{H}^{\text {top }}}{\partial t}
$$

Let us point out that is exactly at eq(3) that the presence of compact holes on $\mathbb{R}^{3}$ alters significantly the usual analysis to write the equations for the electromagnetic potentials. On basis of eq(3) one can only conclude that there is an electric field scalar potential $\phi_{E}$ such that

$$
\vec{E}+\frac{1}{c} \frac{\partial \vec{A}_{H}}{\partial t}=\left(-\operatorname{grad} \phi_{E}\right)+\overrightarrow{\hat{\varepsilon}}\left(\phi_{H}, \vec{H}^{\text {top }}\right)
$$

Here $\overrightarrow{\hat{\varepsilon}}\left(\phi_{H}, \vec{H}^{\text {top }}\right)$ is an additional (not yet explicited) nonzero functional of the magnetic potential $\phi_{H}$ and of the harmonic magnetic fields $\vec{H}^{\text {top }}$ as solution of the first order partial differential eq (3) supposed to have non trivial solutions (a further study of this mathematical issue will appears elsewhere $)^{2}$

By substituting eq(4) and eq (2) into eq(1-b) and eq(1-a), one gets our somewhat elec- tromagnetic wave equations for the electromagnetic potentials with "topological sources" in a the "Holed" space $\mathbb{R}^{3}$.

$$
\begin{aligned}
& \left(-\nabla \vec{A}_{H}+\operatorname{grad} \operatorname{div} \vec{A}_{H}\right)+\left(\frac{1}{c^{2}} \frac{\partial^{2}}{\partial t^{2}} \vec{A}_{H}+\frac{1}{c} \frac{\partial}{\partial t} \operatorname{grad} \phi_{E}\right) \\
& =\frac{4 \pi}{c} \vec{J}+\frac{1}{c} \frac{\partial}{\partial t} \overrightarrow{\hat{\varepsilon}}\left(\phi_{H}, \vec{H}^{\mathrm{top}}\right) \\
& -\nabla \phi_{E}-\frac{1}{c} \frac{\partial}{\partial t}\left(\operatorname{div} A_{H}\right)=4 \pi \rho-\operatorname{div} \overrightarrow{\hat{\varepsilon}}\left(\phi_{H}, \vec{H}^{\mathrm{top}}\right)
\end{aligned}
$$

By following the usual protocols, one must fix a gauge on the above set of equations for the Electromagnetic potentials $\left(\vec{A}_{H}, \phi_{E}\right)$ in order to make them mathematically soluble. ${ }^{2}$

We choose thus the well-known and decoupling (and free of mathematical problem) Landau Gauge

$$
\operatorname{div} \vec{A}_{H}+\frac{1}{c} \frac{\partial \phi_{E}}{\partial t}=0
$$

After implementing this gauge fixing, one gets the dynamical equations satisfied by the Electromagnetic potentials in the gauge $\mathrm{eq}(6)$.

$$
\begin{array}{r}
\Delta \vec{A}_{H}-\frac{1}{c} \frac{\partial^{2} \vec{A}_{H}}{\partial t^{2}}=-\frac{4 \pi}{c} \vec{J}_{E}-\frac{1}{c} \frac{\partial}{\partial t} \overrightarrow{\hat{\varepsilon}}\left(\phi_{H}, \vec{H}^{\mathrm{top}}\right) \\
\Delta \phi_{E}-\frac{1}{c^{2}} \frac{\partial^{2} \phi_{E}}{\partial t^{2}}=-4 \pi \rho+\operatorname{div} \overrightarrow{\hat{\varepsilon}}\left(\phi_{H}, \vec{H}^{\mathrm{top}}\right)
\end{array}
$$

We note the presence of news effectives electrical current and electrical charge densities on the eq(7-a) and eq(7-b) due to the ( (harmonic" pieces of the magnetic field.

Let us stress again that the topological harmonic magnetic configuration $\vec{H}^{\text {top }}$ on the Helmoholtz decomposition eq(2) must be given as boundary conditions and must be suppose fully explicited.

As a result of eq(1-c), now with a non trivial magnetic source $\vec{J}_{m}$ for sake of generality, one obtains that:

$$
\operatorname{div}\left(\nabla \times \vec{A}_{H}+\operatorname{grad} \phi_{H}+\vec{H}^{\text {top }}\right)=\operatorname{div} \vec{J}_{n}
$$


As a result one has

$$
\phi_{H}=-\Delta^{-1}\left(\operatorname{div}\left(\vec{H}^{\text {top }}+\vec{J}_{m}\right)\right)
$$

Where $\Delta^{-1}$ is the Green function of the Laplacean operator on the $\mathbb{R}^{3}$ with compac holes inside, certainly a highly non trivial problem even on the "Riemann surface" case of $\mathbb{R}^{2} .^{2}$

Another useful relation is obtained from eq(3) and the unrotationality of $\vec{H}^{\text {top }}$

$$
\nabla \times \nabla\left(\vec{E}+\frac{1}{c} \frac{\partial \vec{A}}{\partial t}\right)=0
$$

which means that again under the imposed condition $\operatorname{rot}\left(\vec{H}^{\text {top }}\right) \equiv 0$, the following equation

$$
-\Delta\left(\vec{E}+\frac{1}{c} \frac{\partial \vec{A}}{\partial t}\right)+\operatorname{grad}\left(\operatorname{div}\left(\vec{E}+\frac{1}{c} \frac{\partial \vec{A}}{\partial t}\right)\right)=0
$$

It is now worth call attention for the standard gradient solution of eq(11)

$$
\vec{E}+\frac{1}{c} \frac{\partial \vec{A}}{\partial t}=-\left(\operatorname{grad} \phi_{E}\right)
$$

Note that under the solution eq(12) the usual dynamical equations for the electromagnetic potentials eq(7-a) and eq(7-b) with $\overrightarrow{\hat{\varepsilon}}\left(\phi_{H}, \vec{H}^{\text {top }}\right) \equiv 0$, are obtained.

Let us thus point at that on the quantum mechanical world the first basic equations are the potential wave equations eq $(5-\mathrm{a})$ and eq $(5-\mathrm{b})$ with $\overrightarrow{\hat{\varepsilon}}\left(\phi_{H}, \vec{H}^{\text {top }}\right) \equiv 0$, with the meaning that on the quantum world, the space is fully $\mathbb{R}^{3}$, without any non trivial differ- ential topological structure immersed on it for mathematical consistency of its dynamical equations and the physical assumptions in Quantum Mechanicsnon relativistic case. As an pedagogical comment we write the path integral expression for the mild point rule Feynman propagator of a charged particle in the presence of a magnetic constant, but Gaussian random potential $\tilde{A}_{H}$ and zero magnetic field $\vec{H}$ :

$$
\begin{aligned}
& G\left(\vec{x}_{1}, \vec{x}_{2}, T,\left[A_{H}\right]\right)=\int_{\vec{x}(0)=\vec{x}_{1}}^{\vec{x}(T)=\vec{x}_{2}} D^{F}[\vec{x}(\sigma)] \exp \left(-\frac{i}{\hbar} \int_{0}^{T} \frac{1}{2} m\left(\frac{d \vec{x}}{d \sigma}\right)^{2}(\sigma) d \sigma\right) \\
& \times \exp \left\{-\frac{i e}{2 c \hbar} \int_{0}^{T} d \sigma\left[\frac{d x^{i}}{d \sigma} \mathcal{E}_{i j s}\left(\frac{\partial}{\partial x^{j}} B_{A}^{s}\left(x^{\ell}\right)\right)\right](\sigma)\right\}
\end{aligned}
$$

We can see that the averaged Feynman propagator with $\vec{x}==\left(x_{1}, x_{2}, x_{3}\right)$ and $\vec{y}=\left(y_{1}, y_{2}, y_{3}\right)$ is explicitly given by

$$
\begin{aligned}
E_{A}\left\{G\left(\overrightarrow{\mathrm{x}}, \overrightarrow{\mathrm{y}} t,\left[\vec{A}_{H}\right]\right)\right\}=G_{0}\left(\left(x_{1}, x_{2}\right) ;\left(y_{1}, y_{2}\right), T\right) \\
\quad \times E_{A_{3}}\left\{e^{-\frac{i e}{c \hbar}} A_{3} \int_{0}^{T} d \sigma \frac{d z}{d \sigma}\right\} \\
=G_{0}\left(\left(x_{1}, x_{2}\right) ;\left(y_{1}, y_{2}\right), T\right) \times \\
\left(\sqrt{\pi \mu^{2}}\right) \exp \left\{-\left[\frac{1}{2} \mu^{2} \frac{e^{2}}{c^{2} \hbar^{2}}\left(x_{3}-y_{3}\right)^{2}\right]\right\}
\end{aligned}
$$

Here $G_{0}\left(\left(x_{1}, x_{2}\right),\left(y_{1}, y_{2}\right), T\right)$ denotes the free propagation on the plane and $\mathbb{R}^{2}$.

It is curious that the "damped" Ahronov-Rhom effect predicts by eq(12) means dissipation on quantum mechanics by a zero classical magnetic field but with a random constant vector potential and nonzero Helmoholtz potential vector, a new physical quantum phenomena predicts by us.

Finally we point out for sake of comparasion with eqs $(7 a-7 b)$ that the Maxwell Equations eq(1-a)-eq(1-d) take the following form, if one uses directly the Helmoholtz theorem for the electric and magnetic fields

$$
\begin{aligned}
& \vec{E}=\left(\nabla \times \vec{A}_{E}\right)+\left(\operatorname{grad} \phi_{E}\right)+\vec{E}^{\text {top }} \\
& \vec{H}=\left(\nabla \times \vec{A}_{H}\right)+\left(\operatorname{grad} \phi_{H}\right)+\vec{H}^{\text {top }}
\end{aligned}
$$

The set of our proposed news equations for the Electromagnetic Field for a Holed space $\mathbb{R}^{3}$ takes the form (plus suitable boundary conditions) $)^{(*)}$

$$
\begin{aligned}
\Delta \phi_{E} & =4 \pi \rho-\operatorname{div} \vec{E}^{\text {top }} \\
\Delta \phi_{H} & =-\operatorname{div}\left(\vec{H}^{\text {top }}\right)
\end{aligned}
$$

$-\Delta \vec{A}_{H}+\operatorname{grad}\left(\operatorname{div} \vec{A}_{H}\right)=\frac{4 \pi}{c} \vec{J}+\frac{1}{c} \frac{\partial}{\partial t}\left(\operatorname{rot} \vec{A}_{E}+\operatorname{grad} \phi_{E}+\vec{E}^{\text {top }}\right)(17-\mathrm{c})$

$$
-\Delta \vec{A}_{H}+\operatorname{grad}\left(\operatorname{div} \vec{A}_{E}\right)=\frac{1}{c} \frac{\partial}{\partial t}\left(\operatorname{rot} \vec{A}_{H}+\operatorname{grad} \phi_{H}+\vec{H}^{\text {top }}\right)
$$

Usually one considers the mathematically well define A. Sommerfeld irradiation conditions at the spatial infinity. ${ }^{3}$

In this case $\vec{E}^{\text {top }}$ and $\vec{H}^{\text {top }}$ should be prescribed as boundary conditions for possible well-posedness of the irradiation problem associated to the above written set of PDE's. A complete mathematical and physical study will appears elsewhere in a more detailed paper with applications.

\section{Acknowledgment}

We are thankfull to CNPq for a fellowship and to Professor José Helayel-CBPF and W. Rodrigues-IMEC-UNICAMP.

\section{Conflicts of interest}

Authors declare there is no conflict of interest.

\section{References}

1. Luiz CL Botelho. Methods of Bosonic Path Integrals Representations Random Systems in Classical Physics. New York: Nova SciencePublishers Inc; 2006. p. 363.

2. Luiz C L Botelho. Lecture Notes in Applied Differential Equations of Mathematical Physics. Singapore: World Scientific Publishing; 2008. p. 340 .

3. JD Jackson. Classical Electrodynamics. 2nd edition. New York: John Willey \& Sons; 1975.

4. Luiz C L Botelho. A note on the electromagnetic irradiation in a holed spatial region: A space-time approach. Modern Physics Letters B. 2017;31(5):1750039. 